\title{
Résultat de quelques recherches sur le bolide du 29 Octobre 1857.
}

Par M. F. Petic, Directeur de l'observatoire do Toulouse.

Le bolide du 29 Octobre 1857 , observé à Paris par M. le Maréchal Vaillont et par M. Leverricr, fut également, selon M. l'abbé Moigno, aperçu dans le département de la Sarthe par M. l'abbé Paumard, professeur de sciences au séminaile de Précigné. Grảces aux indications fournies par la note du Cosmos (6 Novembre), aux déterminations effectuées par M. Leverrier (Comptes rendus du 9 Nov.), aux relevés faits par M. le Maréchal Vaillant, avec un empressement dont la science doit lui savoir d'autant plus degré que la multiplicité de ses occupations officielles semblait de nature à détourner plus facilement sa pensée de la constation du phénomène; grâces enfin à l'accord satisfaisant que présentent les observations de Paris; j’ai pu rechercher qu'elles étaient les particularités les plus sallfante de la marche du météore, et j'ai nbtenu les résultats suivants:

Distance du bolide a la terre, yuand il fut apercu de Paris, à $6^{\text {ln }} 6^{\text {n1 }}$ du soir. dans un azimut de 58 degrés

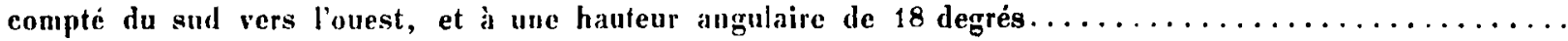

Hllomitien 93, 6

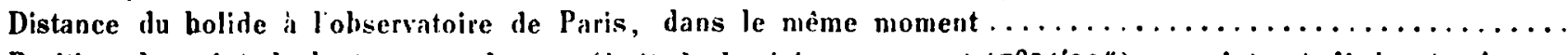

Position du point de la terre au dessus flatitude horéale $=+47^{\circ} 31^{\prime} 20^{\prime \prime}$ ) ce point est situé entre Angers

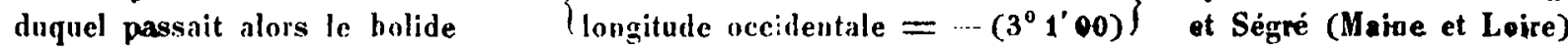

Distance du bolide à la terre, quand il arrivait dans la partie $S_{u} d$ du méridien de Paris, par une hauteur angulaire de 19 degrés, suivi des trois fragments rougeâtres qui s’en étaient détaché ...........

Distance du bolide a l'observatoire de Paris, dans le mème moment $\ldots \ldots \ldots \ldots \ldots \ldots \ldots \ldots \ldots \ldots \ldots$ Position du point de la terre au dessus du- $\left\{\right.$ latitude borćale $\left.=+48^{\circ} 0^{\prime} 10^{\prime \prime}\right\}$ ce point est un peu au Sud de quel passait alors le bolide $\quad$ longitude $\left.=0^{\circ} 0^{\prime} 00\right\}$ Pithiviers (Loiret)

Distance du holide à la terre, quand il fut aperçu le Précigné, à l'ouest-sud-ouest, et à 40 degrés de han-

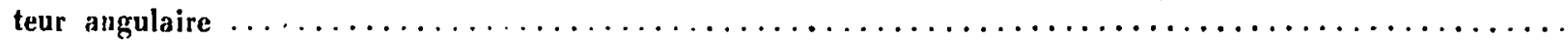

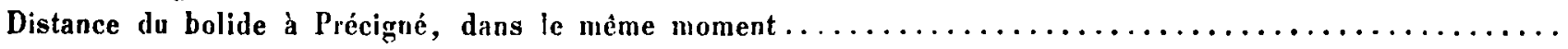

Position du point de la terre au dessus flatifude boréale $\left.=+47^{\circ} 16^{\prime} 40^{\prime \prime}\right\}$ ce polnt est aux environs de

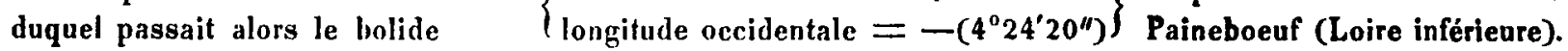

Distance du bolide à la terre, quand il parut se diviser en fragments pour l'observateur de Précigné, à une

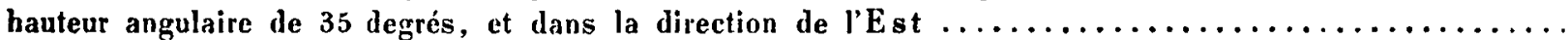

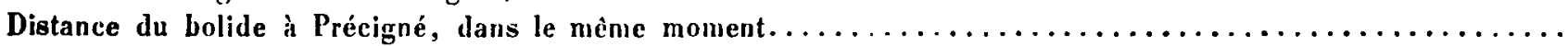

Position du point de la terre au dessus (latitude boréale $\left.=+47^{\circ} 46^{\prime} 40^{\prime \prime}\right\}$ co point est a l'oueat-sud-ouest duquel passait alors le bolide $\quad$ longitude occidentale $\left.=-1^{\circ} 28^{\prime} 50^{\mu}\right\}$ de Veadôme (Lairo et Cher).

Position du point où la trojectoire rencontre la terre et dans le voisinage latitude boréale $=+48^{\circ} 15^{\prime} 30^{\prime \prime}$ (ce point est aux envi-

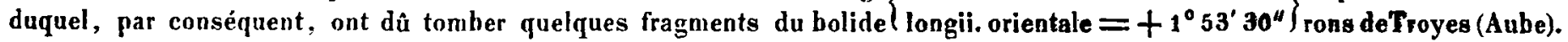

Vitesse apparente déduite de l'observation de Paris dans l'hypothèse d'une durée de 6’5 (moyenoe de l'éraluation 6 à 7 secondes) pour le tenus employé par le bolide à aller de l'un à l'autre des deux points de la trojectoire apparente, qui ont été désignés à dessus . 37122 mètres.

Cette vitesse s'accorde tris convenablement avec l'observation de M. l'ahbé Paumard qui évalue à 10 ou 12 secondes la durée totale du phémonène, en faisant remarquer tontefois que les fragments dans lesquels s'est divisé le bolide ont eux mèmes brillé pendant quelques secondes de temps. Car pour obtenir par l'ohservation de Précigné la mème vitesse, exactement, que par l'observation de Paris, il suffit de supposer à la première partie du phénomène, à celle qui sépare les instants du passage du bolide par les deux points de la trojectoire apparente (à Précigné), emplnyés dans les calculs précédents, une durée de $6^{\circ} 42$; ce qui laisserait, en effet, un excédant de 3 à 4 secondes, conformément aux indications de M. Paumard, pour la durée du temps pendant lequel ont brillé les fragments.

En tenant compte du double mouvement (rotation et translation) de la terre, j’ai trouvé en outre:

Vitesse géocentrique du bolide ... 37386,95

Vitesse héliocentrique ....... 56197,76

D’où j'ai déduit pour les orbites décrites par le bolide soit autour de la terre soit autour du soleil, au moment de 
l'apparition, des orbites largement hyperboliques, puisque l'excentricité de l'une serait égale à 21 et celle de l'autre à 2 environ. J'ajoute que l'influence de la terre avait peu modifié l'orbite primitive du bolide, celle dans laquelle if se mouvait autour du woll sant d'etre boumis a cette influence; car elle n'avait tlevé que de 1,80 1,96 le chiffre de l'excentricite. Et quand à la résistance de l'air, il ne m'a pas pars qu'il y eut istérét à cbercher sa valeur, puisqu'en diminuant la vitesse du bolide, elle n'avait fait que rendre moins sensibles les résaltats obtenus et qu'ajouter, par conséqueat, une probabilité nourelle à ces résultats. Jusqu'à quel point est - il permis de regarder comme démontrées les conséquences qui on d'́conlent? Je n'oserais prendre sur moi de le décider; et je laisse à ceux que ces curieuses questions peuvent intéresser, le soin de se créer une opinion à cet égard, en me bornant à faire remarquer que pour ramener l'orbite primitive à otre elliptique, il faudrait réduire la vitesse apparente donné par l'observation de $\mathbf{M}$. Leeverrier, de 37 mille à 22 mille mètres environ; ce qui parait bien exagéré. L'on pourrait, il est vrai, supposer qu'avant son arrivee dans le volsinage de la terre, le bolide a eu ses éléments radicalement modifiés par quelques uns des corps du système solaire; car les orbites résultant d'observations nécessairement imparfaites, no sont jamais ussez précises pour permettre de suive la marche du météore ì partir de distances un peu considérables et, par conséquent aussi, pour permettre de déteminer les perturbations qu'il a pu éprouver en venant vers nous.

Néaumoins, on doit le reconnaitre; en égard au peu de place que les corps célèste oceupent dans l'immensité de l'espace, il semble peu probable qu'un méme bolide soit passé plusieurs fois, pendant la durée de sa marche autour du soleil, assez près des planètes pour être dérangé par elles, comme il l'a été par la terre.

Le bolide du 29 Octobre 1857 serait done venu, d'après cela, ainsi que certaines comètes, de la région des étoiles. Mais, sous un point de vue tout spécial, plus utile encore que ces comètes aux progrés de la philosopbie naturelle, il nous aurait donné par la chute sur la terre, s'il eut pu être retrouvé, de curieuses révélations sur la constitution matérielle des régions si éloignées d'où la lumiêre elle-même, malgré son étonnante vitesse, met des années et nême des siècles à nous parvenir. Malheureusement, il est rare de rencontrer les circonstances exceptionelles, qui, parmi de nombreux fragments dispersés et perdus, out fait retrouver, dernièrement, les deux aréolithes d'Ausson et de Clarac; et la plupart de ceux qui tombent sans doute sur la Terre, restent inutiles à la science. Dans tous les cas, de quelque manière qu'on envisage les recherches précédentes, les aspects si variés et si intéressants que présente l'étude des holides me paraissent bien faits pour appeller l'attention les efforts des astronomes sur un sujet trop délaisé jusqu'ici.

Au reste, soit que le bolide du 29 Octbr. 1857 fut venu de la région des étoiles, soit qu'il eut au contraire circulb de tout temps aytour du soleil, il pourra devenir, un jour, utile de connaitre la valeur numérique des éléments fournis par les ohservations. Sous les réserves faites plus haut an point de vue de l'exactitude de ces éléments qui ne peuvent ètre considérés que comme des éléments limites et non comme des éléments rigoureusement vrais, voici donc ce que j’ai trouvé:

Eléments de l'orbite hyperbolique daos la quelle se mouvait le bolide autour, de la terre quand il fut aperçu.

Excentricité $\ldots \ldots \ldots \ldots \ldots \ldots \ldots \ldots=20,89814$

Demi grand axe ..............

Distance périgée . . . . . . . . . . . $=6219$ Kilomètres

AR du noeud ascendant sur l'équatenr. $=235^{\circ} 26^{\prime} s^{\prime \prime}$

Inclinaison sur l'équateur .........

Sens du mouvement géocentrique en AR direct. Passage au périgée:

le 29 Octobre 1857 à $6^{\mathrm{h}} 6^{\mathrm{n}} 46^{\mathrm{s}} 71$ du soir (temps moyen de Paris)

Eléments de l'orbite hyperbolique que le bolide aurait décrite autour du soleil, en vertu de la vitesse dont il était animé au noment de l'observation, si la terre n'eut pas existé; c'est-à-dire éléments de l'orbite troublé par la terre:

Excentricité ............... $=1,9586285$

Demi grande axe ............ $=-0,7162482$,

Distance péribélie............

AR du noeud ascendant sur l'équateur $=304^{\circ} 34^{\prime} 20^{\prime \prime}$

Inclinaison sur l'équateur .........

Sens du mouvement héliocentrique en AR direct. Passage au périhélie le 4 Oct. 1857 à $3^{\mathrm{h}} 32^{\mathrm{m}} 15^{\mathrm{s}}$ du soir (ha.d.P.)

*) La distance moyenne de la terre au soleil étant t'unite.

Eléments de l'orbite hyperbolique, dans la quelle we mouvait le holide autour du soleil, avant d'etre soumis à l'influence de la terre:

Excentricité ...............

Demi grand axe ............. $=-0,8436038$ )

Distance périhélie ............ 0,6734830$\}^{*}$

AR du woeud ascendant sur l'équateur $=313^{\circ} 20^{\prime} 00^{\prime \prime}$

Inclinaison sur l'équateur ........ $=13^{\circ} 53^{\prime} 40^{\prime \prime}$

Sens du mouvement héliocentıique en AR direct. Pass. au périhélie le 30 ct. 1857 a $6^{\mathrm{h}} 10^{\mathrm{m}} 40^{\mathrm{s}}$ du matin (t. m.d.P.)

*) la distance moyenne de la terre an soleil etant 'umité.

Toulouse le 5 Jaurier 1859 .

F. Petit, Directenr de l'observ. de Toulouse. 